\title{
Fixator-assisted Technique Enables Less Invasive Plate Osteosynthesis in Medial Opening-wedge High Tibial Osteotomy: A Novel Technique
}

\author{
Dong Hoon Lee MD, PhD, Keun Jung Ryu MD, \\ Hae Hwa Kim MD, PhD, Sahyun Soung MD, \\ Soowan Shin BS
}

Published online: 29 May 2015

(C) The Association of Bone and Joint Surgeons (B) 2015

\begin{abstract}
Background Opening-wedge high tibial osteotomy is a well-established procedure in the management of medial osteoarthritis of the knee and correction of proximal tibia vara. Recently, surgical approaches using less invasive plate osteosynthesis have been used with the goal of minimizing complications from more extensive soft tissue exposures. However, to our knowledge, less invasive fixator-assisted plate osteosynthesis has not been tested in the setting of opening-wedge high tibial osteotomy.
\end{abstract}

Each author certifies that he or she, or a member of his or her immediate family, has no funding or commercial associations (eg, consultancies, stock ownership, equity interest, patent/licensing arrangements, etc) that might pose a conflict of interest in connection with the submitted article.

All ICMJE Conflict of Interest Forms for authors and Clinical Orthopaedics and Related Research ${ }^{\circledR}$ editors and board members are on file with the publication and can be viewed on request. Clinical Orthopaedics and Related Research ${ }^{\mathbb{R}}$ neither advocates nor endorses the use of any treatment, drug, or device. Readers are encouraged to always seek additional information, including FDA-approval status, of any drug or device prior to clinical use. Each author certifies that his or her institution approved the human protocol for this investigation, that all investigations were conducted in conformity with ethical principles of research, and that informed consent for participation in the study was obtained.

This work was performed at CHA Bundang Medical Center, CHA University, Sung-nam, Korea.

D. H. Lee ( $\square)$, K. J. Ryu, S. Soung

Division of Orthopaedic Surgery, Severance Children's Hospital,

Yonsei University College of Medicine, Yonsei University,

Seoul 120-752, Korea

e-mail: drdonghoon@yuhs.ac; orthopaedee@ naver.com

H. H. Kim

Department of Orthopaedic Surgery, CHA Bundang Medical

Center, Sung-nam, Kyungki-do, Korea
Questions/purposes The purposes of this study were (1) to assess the complications associated with use of a fixatorassisted less invasive plate osteosynthesis technique to stabilize an opening-wedge high tibial osteotomy in the treatment of proximal tibial vara; and (2) to evaluate the ability of this technique to achieve correction of the proximal tibial deformity and achieve osseous union.

Methods From June 2011 to June 2013, a total of 157 limbs in 83 patients who underwent fixator-assisted high tibial osteotomy for (1) idiopathic genu vara; or (2) osteoarthritis of the knee with proximal tibia vara were initially enrolled. Of these, eight limbs (5\%) were excluded on the way; thus, 149 limbs in 77 patients were evaluated. During the period in question, no other techniques were used for proximal tibial osteotomy. The surgical procedures included less preparation of soft tissue, proximal tibial osteotomy, application of a temporary external fixator, correction of alignment, and final fixation with the help of an external fixator. Complications were assessed by chart review and the alignment in both coronal and sagittal planes was compared pre- and postoperatively. Radiographic review to confirm osseous union and alignment was performed by two of the authors not involved in clinical care of the patient. Delayed union was described as union occurring later than 4 months.

Results Thirty limbs out of 149 tibiae (20\%) showed complications, all of which were resolved without leaving

S. Shin

Department of Biological Sciences, University of Southern California, Los Angeles, CA, USA 
any sequela. Twenty-seven limbs out of 149 limbs (18\%) showed lateral cortical hinge fracture and three limbs out of 149 limbs (2\%) showed soft tissue complications (two superficial infections, one wound hematoma). The overall completeness of reaching the target correction was excellent. In the coronal plane, the difference between the amount of real correction and the amount of target correction was $0.3^{\circ} \pm 0.7^{\circ}(\mathrm{p}<0.001)$. In the sagittal plane, the difference between pre- and postoperative posterior proximal tibial angle was $-0.1^{\circ} \pm 0.2^{\circ}(\mathrm{p}<0.001)$. All osteotomies healed before 4 months.

Conclusions Fixator-assisted high tibial osteotomy is a valid option for medial opening-wedge high tibial osteotomy, which enables less invasive surgery with excellent coronal/sagittal/rotational alignment control. However, future studies should compare this approach with other approaches for proximal tibial osteotomy to ascertain whether indeed this procedure is less invasive or more reliable.

Level of Evidence Level IV, therapeutic study.

\section{Introduction}

Opening-wedge high tibial osteotomy is a well-established procedure in the management of medial osteoarthritis of the knee and correction of proximal tibia vara. Conventional opening-wedge high tibial osteotomy is associated with complications including implant breakage, lateral cortical fracture, fracture of the lateral tibial plateau, increased posterior tibial slope, delayed union or nonunion, delayed would healing, and deep infection $[2,4,9,15,25$, 26]. Since angle-stable locking plates were introduced, implant-related complications have been reduced considerably [2, 12]. However, soft tissue problems, delayed union or nonunion, and deep infection still are reported to be relatively frequent $[9,12,15,23,25]$ in conventional opening-wedge high tibial osteotomy, which may result from extensive soft tissue exposure to protect posterior neurovascular structures or to apply the plate while maintaining a distractor in place during the surgery.

Recently, surgical approaches using less invasive plate osteosynthesis have been used in various fracture surgeries to diminish soft tissue complications and promote biologic bone healing $[3,17,28]$. Although the less invasive technique has shown to have fewer soft tissue complications, faster union, and better cosmesis [6, 17, 19, 21, 28], it has never been devised to use in opening-wedge high tibial osteotomy.

The purposes of this study were (1) to assess the complications associated with use of a fixator-assisted plate osteosynthesis technique to stabilize an opening-wedge high tibial osteotomy in the treatment of proximal tibial vara; and (2) to evaluate the ability of this technique to achieve correction of the proximal tibial deformity and achieve osseous union.

\section{Patients and Methods}

This was a retrospective study. This study was approved by the institutional review board at our institution. From June 2011 to June 2013, we performed 157 limbs' high tibial osteotomies. All high tibial osteotomies were done with this technique if that was the case during the study period. A total of 157 limbs in 83 patients (74 bilateral, nine unilateral) who underwent fixator-assisted high tibial osteotomy for idiopathic genu vara (proximal tibia vara) or osteoarthritis of the knee with proximal tibia vara of mechanical axis deviations greater than $20 \mathrm{~mm}$ with the following inclusion/exclusion criteria were initially enrolled. Inclusion criteria were (1) correction angle less than $15^{\circ}$; (2) skeletally mature patients; (3) long bone radiographs, which show mechanical femorotibial angle (mFTA) and posterior proximal tibial angle both pre- and postoperatively; (4) postoperative tibial plain radiographs (AP and lateral) with a 1-month interval; and (5) medical records describing detailed postoperative complications. Among them, 149 limbs in 77 patients were eligible, but eight limbs in six patients could not be included because they (1) were lost to followup (four limbs); and (2) had insufficient data in the record (four limbs); three limbs in this group showed no mention on complications in their medical records; however, it was confirmed there were no complications at the most recent outpatient visit. Therefore, a total of 149 limbs (95\%) out of 157 limbs were analyzed for this study. During the period in question, no other techniques were used for proximal tibial osteotomy.

The mean age at the time of surgery was $35 \pm 12$ years and a female-to-male ratio of 79:70 (Table 1). The mean duration of followup was $24 \pm 6$ months (range, 16-40 months). Preoperative mFTA was $7^{\circ} \pm 2^{\circ}$ varus.

Table 1. Demographics of the patients who underwent fixator-assisted high tibial osteotomy

\begin{tabular}{ll}
\hline Demographic data & $\begin{array}{l}\text { Total } 149 \\
\text { tibiae }\end{array}$ \\
\hline Age (years) & $35 \pm 12$ \\
Sex (female:male) & $79: 70$ \\
Body mass index $\left(\mathrm{kg} / \mathrm{cm}^{2}\right)$ & $23 \pm 3$ \\
Smoking history (yes:no) & $45: 104$ \\
Duration of followup (months) & $24 \pm 6$ \\
Preoperative mechanical femorotibial angle & $7 \pm 2$ varus \\
\hline
\end{tabular}

Values are expressed as means with ranges in parenthesis without "smoking history". 
In an earlier study, we evaluated the simultaneous medial opening-wedge high tibial osteotomy and modified Maquet procedure for combined medial compartmental and patellofemoral osteoarthritis of the knee [16]. Indications and surgical techniques were different between the studies, and none of the patients in the current report were included in that earlier report.

\section{Surgical Technique and Postoperative Protocol}

\section{Preparation of Soft Tissue}

All surgeries were performed by one senior surgeon (DHL). All patients were under general anesthesia and were operated on in the supine position on a routine operating table. First, a $2.5-\mathrm{cm}$ oblique incision is made midway between the center of the tibial tuberosity and the posteromedial border of the proximal tibia, starting from $1 \mathrm{~cm}$ below the upper margin of the pes anserinus tendon extending proximally in parallel with the posteromedial border of tibia (Fig. 1A-B). Careful dissection follows to

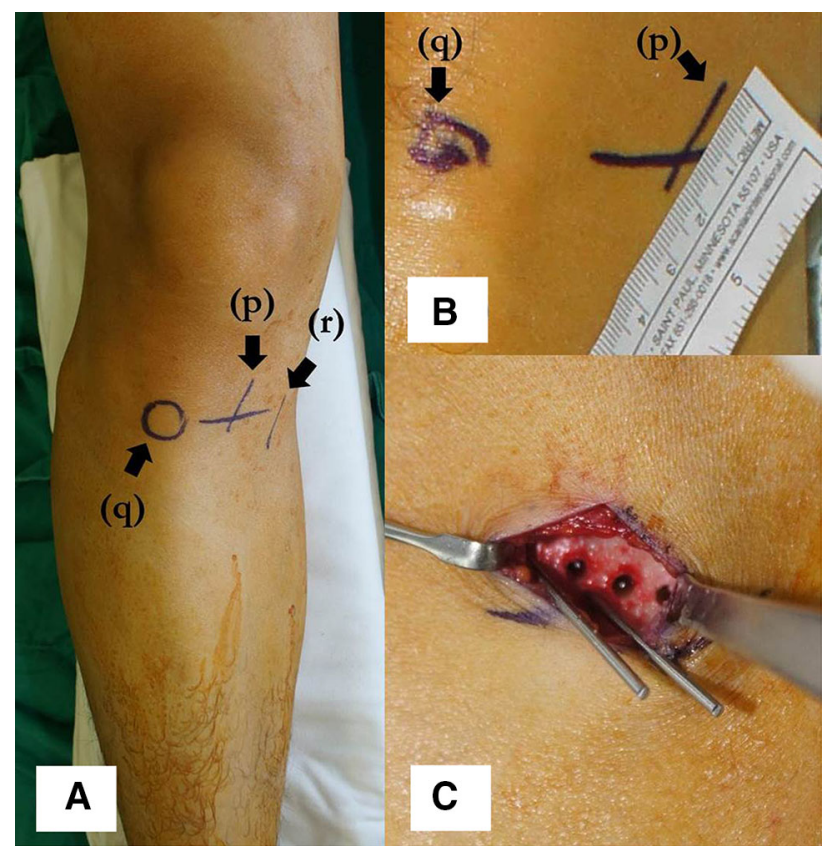

Fig. 1A-C (A) This intraoperative clinical image shows the patient's operated leg at the early stage of fixator-assisted high tibial osteotomy. A $2.5-\mathrm{cm}$ oblique incision is made midway between the center of the tibial tuberosity and the posteromedial border of the proximal tibia. $\mathrm{p}=2.5-\mathrm{cm}$ oblique incision; $\mathrm{q}=$ center of the tibial tuberosity; $r=$ posteromedial border of the proximal tibia. (B) The oblique skin incision starts from $1 \mathrm{~cm}$ below the upper margin of the pes anserinus tendon and extending proximally in parallel with the posteromedial border of the tibia. $\mathrm{p}=2.5-\mathrm{cm}$ oblique incision; $\mathrm{q}=$ center of the tibial tuberosity. (C) Multiple drill holes are made along the superior margin of the two guidewires before osteotomy. expose the deep fascia over the pes anserinus and anterior margin of superficial fibers of the medial collateral ligament (MCL) (Fig. 2). After a sharp L-shaped incision is made along the anterior border of the MCL and upper margin of the semitendinosus tendon, the MCL is raised and peeled off with a periosteal elevator. The amount of subperiosteal release of the MCL depends on the amount of correction planned. The first guidewire is inserted toward a "safe zone" [13] with a starting point of $3 \mathrm{~mm}$ beneath the upper margin of the semitendinosus tendon and anterior one-third of the medial surface of the tibia. The second guidewire is inserted from $1 \mathrm{~cm}$ posterior to the first guidewire completely parallel with the first one under fluoroscopic guidance so that the osteotomy plane could be parallel with the plane of the posterior tibial slope. Then, a Mini-Hohmann retractor (DePuy, Synthes, West Chester, PA, USA) is inserted underneath the MCL, around the posteromedial border of the proximal tibia, and multiple drill holes are made along the superior margin of the two guidewires (Fig. 1C).

\section{Proximal Tibial Osteotomy}

The serial osteotomies are performed with osteotomes and a hammer. The first tibial osteotomy is performed by locating the 10-mm-wide osteotome at the center of medial cortex, flush to and preferably proximal to the guidewire.

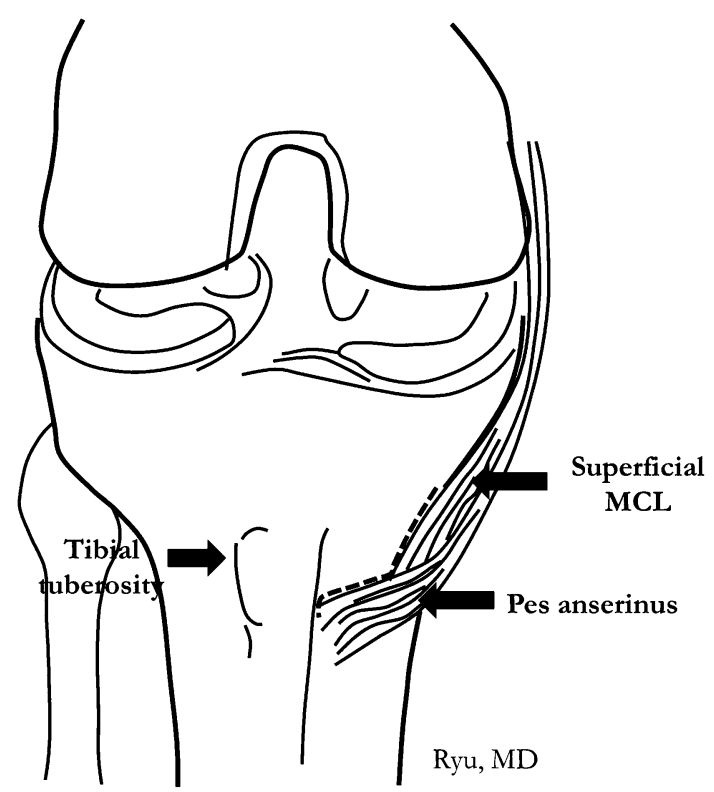

Fig. 2 A schematic illustration shows soft tissue dissection after incision of the skin. A sharp L-shaped incision is made along the anterior border of superficial medial collateral ligament and upper margin of the semitendinosus tendon. These are released subperiosteally. 
Frequently, the osteotomizing procedure is confirmed using a fluoroscopy to ensure that it goes on the right direction with depth (up to $1 \mathrm{~cm}$ medial to the lateral cortex of tibia). The second osteotomy is for the posterior cortex. The knee is flexed to $70^{\circ}$ to $90^{\circ}$ to release posterior neurovascular structures from the posterior cortex. Then the osteotomy is performed using the same osteotome from the posteromedial to posterolateral border in sequence to get a complete transverse cut of the posterior cortex (Fig. 3A). In each step, the depth of the osteotomy can be confirmed using fluoroscopy. With experience, it can be done safely without fluoroscopy, because the surgeons can feel the exact time point of the complete cut of the posterior cortex. The third osteotomy is for the anterior cortex to make a biplanar osteotomy. The direction of osteotomy can be either proximal or distal to the tibial tuberosity (Fig. 3B). We do not use powered oscillating saws to minimize heat injury to the surrounding bone.

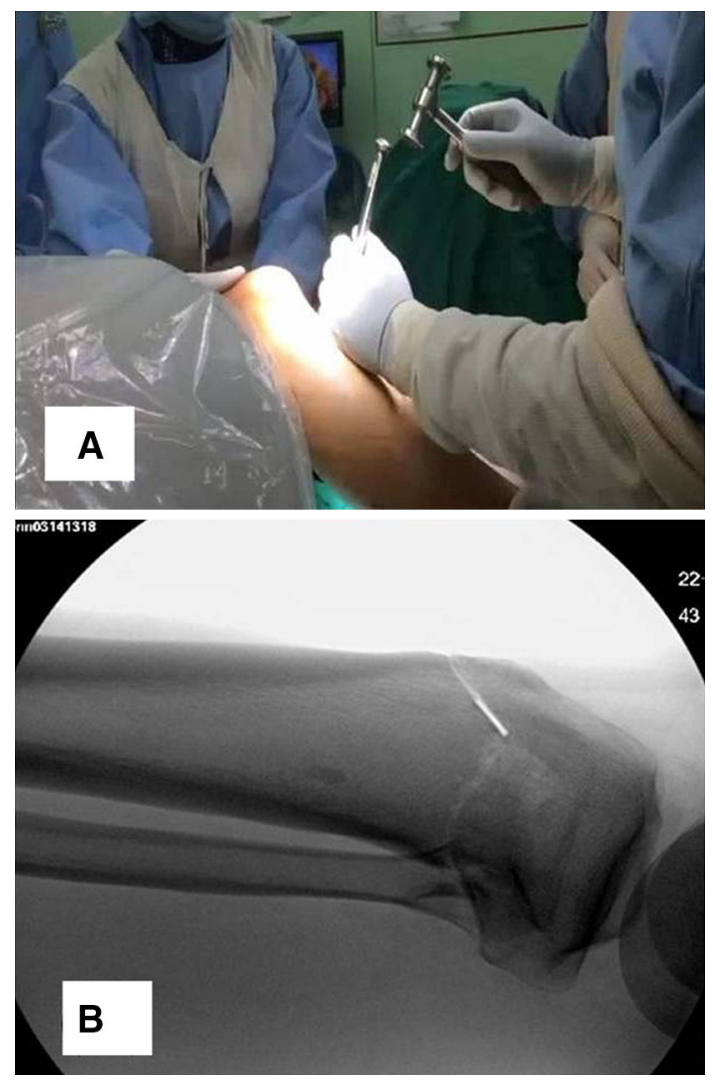

Fig. 3A-B (A) Proximal tibial osteotomy is performed. When performing osteotomy for the posterior cortex, the knee is flexed to $70^{\circ}$ to $90^{\circ}$ to release posterior neurovascular structures from the posterior cortex and the osteotomy is performed from the posteromedial to posterolateral border. (B) When proximal tibial osteotomy is performed, the osteotomy for the anterior cortex can be either proximal or distal to the tibial tuberosity. This $\mathrm{C}$-arm image shows anterior cortical osteotomy distal to the tibial tuberosity.

\section{Application of the External Fixator}

After complete biplanar osteotomy, two 6-mm half pins are inserted at the medial aspect of the proximal tibia for temporary fixation. The proximal half pin is located 1 to $1.5 \mathrm{~cm}$ distal to the joint line and parallel in line with the posterior cortex of the tibial shaft (Fig. 4A). It should be advanced to $1 \mathrm{~cm}$ medial to the lateral cortex and parallel to the knee. The distal pin is located just anterior to the posterior cortex of the tibia. Posteriorly located half pins are important in this technique, because it enables plating providing space at the medial surface of the tibia and helps maintain the posterior tibial slope. These half pins can also be used to check on the changes in rotational alignment after varus correction (Fig. 4B). After confirming proper location of the half pins, the leg is fully extended. The wedge-shaped spreader (TomoFix Bone Spreader; DePuy, Synthes) is then gently inserted into the osteotomy site and advanced $1 \mathrm{~cm}$ medial to the lateral cortex. Using this wedge-shaped spreader, the gap is widened correcting varus deformity. This procedure should be performed slowly and carefully to prevent fracture at the lateral cortex and lateral tibial plateau. During this procedure, keeping the knee fully extended is important to avoid unwanted changes in posterior tibial slope. After confirming that the gap is widened easily without forceful manipulation, the wedge-shaped spreader can be removed. A $15-\mathrm{cm}$ carbon rod with three Schanz pin clamps (Khan Medical, Seoul, Korea) is connected to the two half pins on the tibia.

\section{Correction of Alignment and Fixation}

A bone spreader forceps (Bone Spreader, soft lock; DePuy, Synthes) is engaged into the posteromedial corner of the gap to spread the osteotomy. The spreader is locked when sufficient varus correction is obtained grossly (Fig. 5A). Precise correction with fine tuning to accomplish preoperatively planned alignment is possible using the external fixator and bone-holding forceps (self-centering boneholding forceps with a speed lock; DePuy, Synthes; Fig. 5B-D). Several methods have been introduced to check the degree of alignment correction. Although it depends on a surgeon's preference, we prefer the grid method (Fig. 5). The target alignment was $5 \mathrm{~mm}$ of mechanical axis deviation for idiopathic genu vara and $\mathrm{Fu}-$ jisawa point [10] for osteoarthritis of the knee. The temporary external fixator is firmly fixed when the corrected alignment is satisfactory. Keeping the fixator in place, the gap is filled with hydroxyapatite or ß-tricalcium phosphate graft (Bongros ${ }^{\circledR}$; CGBio, Seongnam-si, South Korea) or allograft cancellous chip bone graft (OSG-DualPor ${ }^{\circledR}$; OssGen, Gyungsan-si, South Korea) (Fig. 6A). Then, a plate (TomoFix ${ }^{\circledR}$, TomoFix Medial High Tibial Plate; DePuy, 

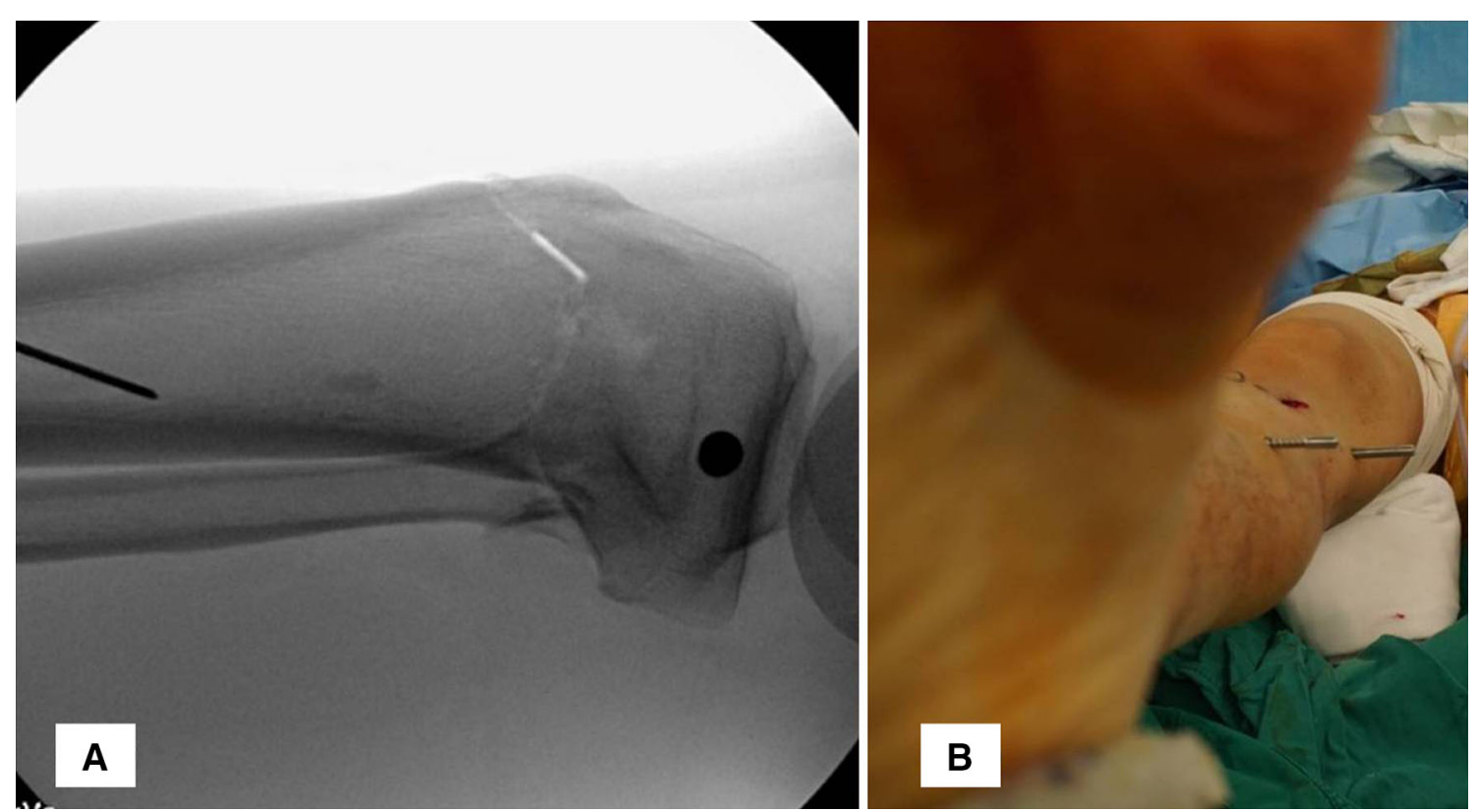

Fig. 4A-B (A) Location of two half pins is shown. Posteriorly located half pins are important, because they enable plating providing space at the medial surface of the tibia and help maintain posterior tibial slope. (B) The two half pins for application of external fixation can also be used to check the changes in rotational alignment.

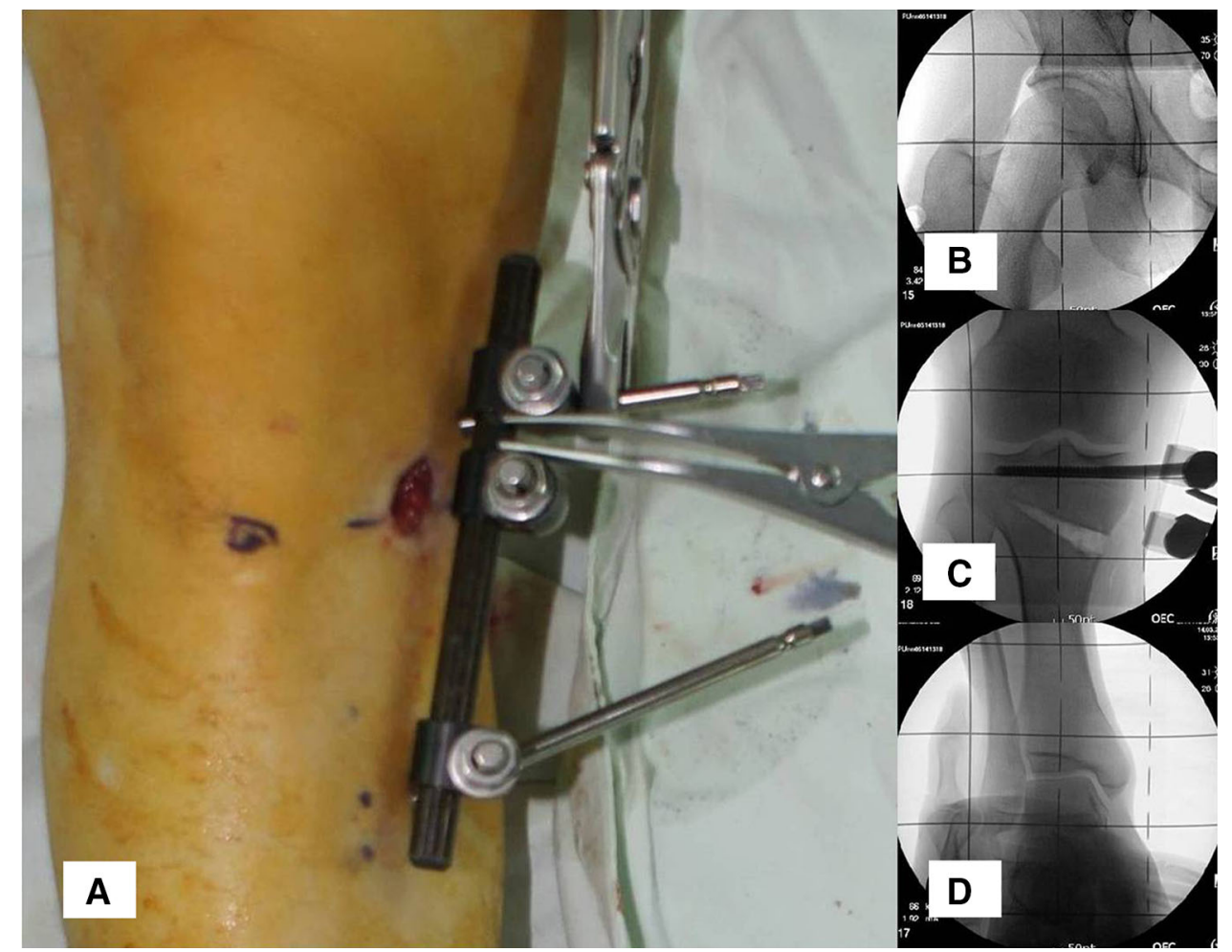

Fig. 5A-D (A) Correction of alignment is performed. Precise correction with fine tuning to accomplish preoperatively planned alignment is possible using the external fixator and bone-holding forceps. (B) During the alignment correction, the grid method can be useful. A predetermined line on the grid should be at the center of the femoral head of the affected leg. (C) During alignment correction, the grid method can be useful. Once the predetermined line on the grid was set to be at the femoral head center proximally and at the ankle center distally, the surgeon can determine where the same line on the grid should pass through the knee, which can be determined intraoperatively by fine tuning using the external fixator and the bone-holding forceps. (D) During alignment correction, the grid method can be useful. The same line that passed the femoral head center should also pass through the ankle center in the AP view. 

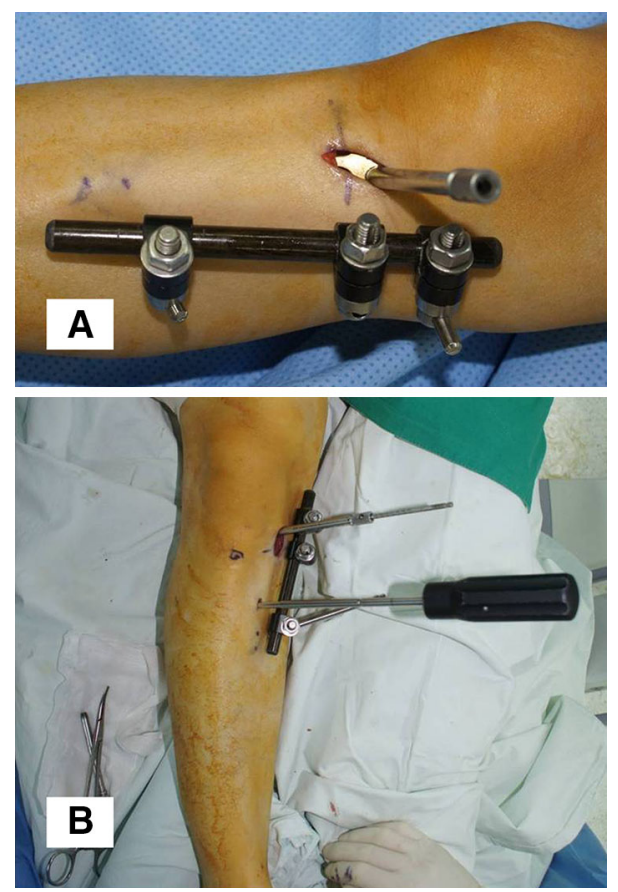

Fig. 6A-B (A) Fixation with a plate $\left(\right.$ TomoFix $^{\circledR}$, TomoFix Medial High Tibial Plate; DePuy, Synthes) is performed. A plate is inserted into the anteromedial surface of the tibia and fixed with $5.0-\mathrm{mm}$ locking screws. (B) The skin incision for the distal half pin can also be used for distal locking screws.

Synthes; Fig. 6B) is inserted into the anteromedial surface of tibia and fixed with 5.0-mm locking screws using a less invasive plating method. The skin incision for the distal half pin can also be used for distal locking screws (Fig. 6). The wound is irrigated and a suction drain is inserted. From postoperative day 1 , gentle active ROM of the knee and full weightbearing are allowed as tolerated. Immediate full weightbearing was allowed for all patients except the patients with lateral cortical hinge fracture. They were allowed to ambulate with crutches 3 weeks postoperatively and then allowed full weightbearing thereafter.

\section{Postoperative Evaluations}

For assessment of complications after fixator-assisted high tibial osteotomy, all patients were followed up regularly (each day during the hospital stay, 3 weeks, 6 weeks, 3 months, 6 months, and 1 year) both clinically and radiographically. All complications related to the index surgery were recorded and categorized into three groups by chart review: (1) soft tissue complications such as superficial/ deep infections, wound hematoma, impaired wound healing, etc; (2) union-related complications such as non- or delayed union; and (3) other complications such as lateral cortical hinge fracture, lateral tibial plateau fracture, hardware failure, symptomatic deep venous thrombosis, complex regional pain syndrome, etc.

For assessment of completeness of reaching target correction, pre- and postoperative standing long bone radiographs were compared. In coronal plane, the amount of target correction planned preoperatively and the amount of real correction obtained postoperatively were compared. In the sagittal plane, pre- and postoperative posterior proximal tibial angle were compared to find any changes in the posterior tibial slope. Radiographic review to confirm osseous union and alignment was performed by two of the authors (SS, JHK) not involved in clinical care of the patients. Delayed union was described as union occurring later than 4 months.

\section{Statistics}

All continuous variables were expressed as mean with a range. Two authors (JHK, SS) participated in radiographic image measurements, and they showed almost perfect interrater agreement (intraclass correlation coefficients, 0.992-0.999) with a measurement error less than $1^{\circ}$ for mFTA, posterior proximal tibial angle, and the amount of real correction. All continuous variables were tested for their normal distribution by Shapiro-Wilk test and were confirmed as such. Paired t-test was used to compare pre- and postoperative posterior proximal tibial angle. A one-sample paired t-test was used to find the difference between the preoperatively planned target correction and the amount of real correction in the coronal plane. The statistical software, $\mathrm{R}$ (Version 2.12 Comprehensive R Archive Network, GNU General Public License) was used for all statistical analysis.

\section{Results}

Among 149 limbs operated on with fixator-assisted high tibial osteotomy, 30 limbs (20\%) showed complications, all of which were resolved without leaving any sequela. Twenty-seven limbs (18\%) showed lateral cortical hinge fracture, all of which were treated successfully without any surgical intervention. Lateral cortical hinge fracture was discovered intra-operatively with help of a C-arm for 20 limbs and postoperatively with plain radiographs for seven limbs. Because we routinely use one cortical screw for plate compression to the bone, there was no limb that showed a gap at the hinge fracture sites. Three limbs (2\%) developed soft tissue complications (two superficial infections, one wound hematoma), which were treated well with administering oral antibiotics and observation of the wound, respectively. There were no other complications (Table 2). 
Table 2. Summary of complications encountered in fixator-assisted high tibial osteotomy

\begin{tabular}{lll}
\hline Complications & $\begin{array}{l}\text { Number } \\
\text { of limbs }(\%)\end{array}$ & Treatment \\
\hline $\begin{array}{l}\text { Soft tissue complications } \\
\text { Superficial infection }\end{array}$ & $2(1.3)$ & $\begin{array}{c}\text { Oral } \\
\text { antibiotics }\end{array}$ \\
Deep infection & $0(0)$ & - \\
Wound hematoma & $1(0.7)$ & Observation \\
Impaired wound healing & $0(0)$ & - \\
Other problems & $0(0)$ & - \\
Union-related complications & $0(0)$ & - \\
Nonunion & $0(0)$ & - \\
Delayed union & & \\
Other complications & $27(18)$ & Observation \\
Lateral cortical hinge fracture & $0(0)$ & - \\
Lateral tibial plateau fracture & $0(0)$ & - \\
Hardware failure & $0(0)$ & - \\
Symptomatic deep venous & & - \\
thrombosis & $0(0)$ & - \\
Complex regional pain syndrome & &
\end{tabular}

Table 3. Completeness of reaching target correction after fixatorassisted high tibial osteotomy

\begin{tabular}{ll}
\hline Radiographic results & $\begin{array}{l}\text { Total } 149 \\
\text { tibiae }\end{array}$ \\
\hline Coronal plane & \\
Amount of target correction (degrees) & $8.0 \pm 0.6$ \\
Amount of real correction (degrees) & $8.1 \pm 0.8$ \\
Mean difference (degrees) & $0.3 \pm 0.7$ \\
p value & $<0.001$ \\
Mean absolute difference $<2^{\circ}$ & $98 \%$ \\
Sagittal plane & \\
Preoperative posterior proximal tibial angle (degrees) & $80 \pm 3$ \\
Postoperative posterior proximal tibial angle (degrees) & $80 \pm 3$ \\
Mean difference (degrees) & $-0.1 \pm 0.2$ \\
p value & $<0.001$ \\
Mean absolute difference $<1^{\circ}$ & $100 \%$ \\
\hline
\end{tabular}

Values are expressed as mean with a SD.

The desired amount of correction in the coronal plane was achieved without causing changes in posterior tibial slope. In the coronal plane, the mean difference between the amount of target correction and the amount of real correction was $0.3^{\circ} \pm 0.7^{\circ}(\mathrm{p}<0.001)$. In the sagittal plane, the mean difference between pre- and postoperative posterior proximal tibial angle was $-0.1^{\circ} \pm 0.2^{\circ}(\mathrm{p}<0.001)$ (Table 3; Fig. 7 A-C). All osteotomies healed before 4 months.

\section{Discussion}

Medial opening-wedge high tibial osteotomy is a common surgical technique for the management of medial compartment knee osteoarthritis and correction of the proximal tibia vara.

However, to our knowledge, a less invasive approach has not been studied in opening-wedge high tibial osteotomy. We aimed to introduce our technique: fixatorassisted high tibial osteotomy, which characterizes less soft tissue exposure, assess the complications associated with this technique, and evaluate the ability to achieve the desired correction and osseous bony union from our series of 149 limbs that underwent the index surgery.

Several limitations should be mentioned. First, most of the patients in this study are relatively young (mean, 35 years; range, 20-67 years) compared with other studies reporting complications in conventional opening-wedge high tibial osteotomy. Because patients in their mid-30s have the potential for better wound healing and better bone quality than older patients, this could affect relatively less complications than other studies $[2,9,15,26]$. Second, this study was based on retrospectively collected data on postoperative complications from chart review, which might decrease the level of evidence of the study. However, we think that it provided helpful information on the advantages of the fixator-assisted technique. Third, this is not a comparative study between fixator-assisted high tibial osteotomy and conventional opening-wedge high tibial osteotomy, which we think is necessary to confirm our results.

In addition, concerns on several methodological biases such as selection bias, transfer bias, and assessor bias should be raised as possible limitations of this study. There is no selection bias because no other surgical techniques were used for proximal tibial osteotomy during the study period. Transfer bias exists in this study, because eight limbs (5\%) out of 157 limbs were dropped out on the way. Among them, four limbs were confirmed to have no complications on their recent visits. The other four limbs were lost to followup; thus, complications of them were still unknown. However, these four limbs occupy only $4 \%$ (four of 149); the transfer bias would be small if any. This should be further clarified. There is no assessor bias because clinical data collection by chart review and radiographic evaluations after the surgery was performed by two of the authors (SS, JHK) not involved in clinical care of the patients and the index surgery.

Although hardware-related complications have decreased after the development of the angle-stable plate, complications such as nonunion, delayed union, or soft tissue problems are commonly encountered [2, 9, 26] (Table 4). There have been several large clinical series 
Fig. 7A-C (A) Preoperative standing long bone radiograph shows proximal tibia vara. The medial proximal tibial angle (MPTA) was $82^{\circ}$ and the mFTA was varus $5^{\circ}$ for both legs. (B) Postoperative standing long bone radiograph shows correction of the proximal tibia vara. The MPTA was $88^{\circ}$ and the mFTA was neutral for both legs. (C) This technique leaves less scar and enables less invasive surgery in medial opening wedge high tibial osteotomy.

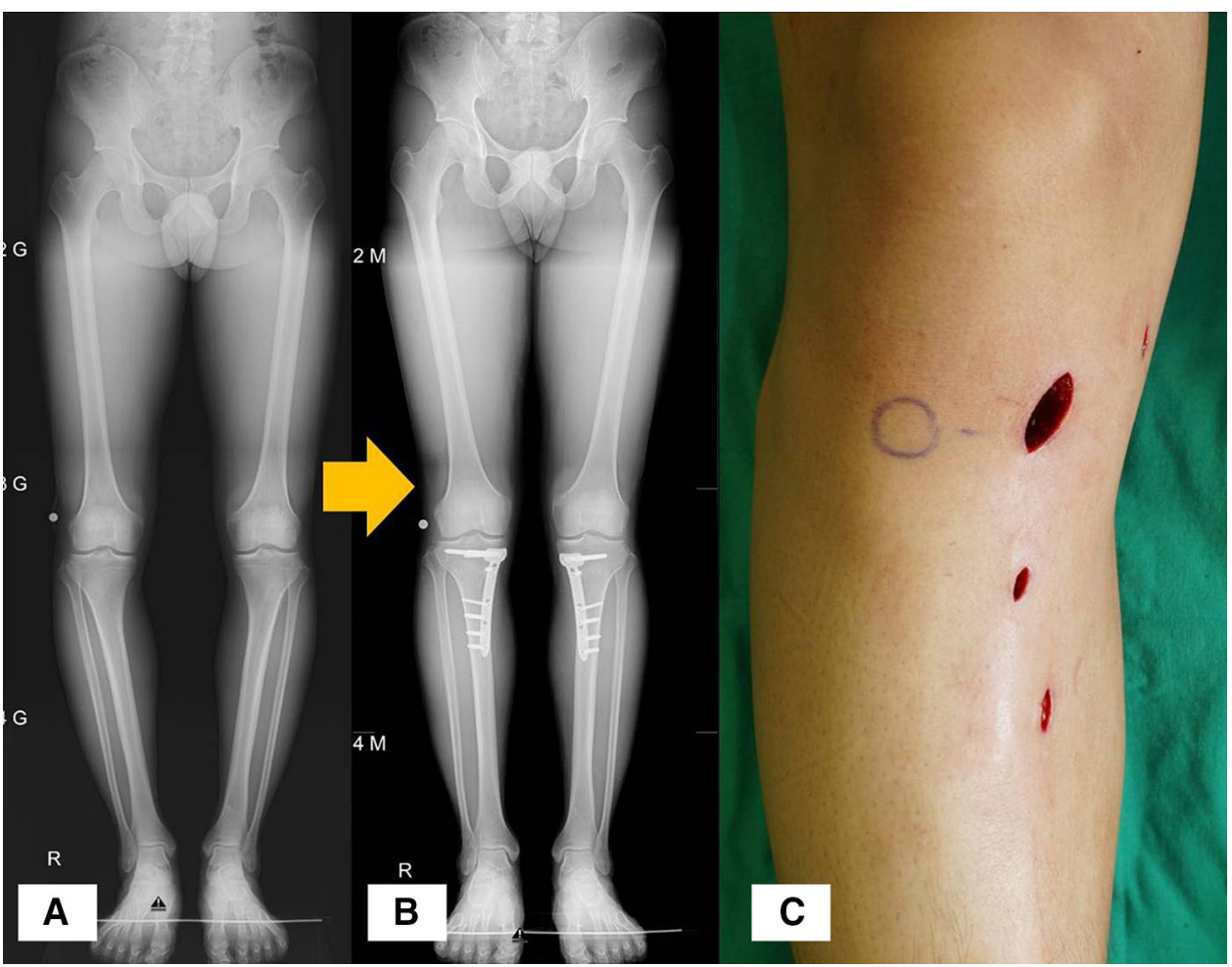

Table 4. Reported complications of angular stable plate (TomoFix ${ }^{\mathbb{R}}$ ) in high tibial osteotomy

\begin{tabular}{|c|c|c|c|c|c|c|}
\hline Study & $\begin{array}{l}\text { Number } \\
\text { of cases }\end{array}$ & Nonunion $(\%)$ & $\begin{array}{l}\text { Soft tissue } \\
\text { problems }(\%)\end{array}$ & $\begin{array}{l}\text { Deep } \\
\text { infection }(\%)\end{array}$ & $\begin{array}{l}\text { Change of tibial } \\
\text { slope }>2^{\circ}(\%)\end{array}$ & $\begin{array}{l}\text { Fracture of } \\
\text { tibial plateau }(\%)\end{array}$ \\
\hline Floerkemeier et al. [9] & 533 & 1.5 & 6 & 2.7 & - & - \\
\hline Meidinger et al. [26] & 186 & 5.4 & - & - & - & - \\
\hline Jung et al. [15] & 92 & 0 & - & 1 & - & 3 \\
\hline Brosset et al. [2] & 51 & 4 & - & 2 & - & - \\
\hline
\end{tabular}

regarding opening-wedge high tibial osteotomy with a conventional surgical technique using the angle-stable plate. Floerkemeier et al. [9] in their multicenter study of 533 segments reported that there was a very small percentage of implant breakage $(0.2 \%)$ but a relatively large percentage of soft tissue problems $(6 \%)$ such as wound hematoma, infection, and impaired wound healing. Brosset et al. [2] reported $6 \%$ of surgical revisions resulting from deep infection and incomplete union. The less invasive plating technique has been developed in an effort to reduce the incidence of surgical complications caused by iatrogenic soft tissue injury and damage to the vascularity of the bone. The less invasive technique offers low risk of nonunion, delayed union, or soft tissue problems [15, 18, 20, 26] by reducing iatrogenic damage to the surrounding soft tissues during the surgery. In this study, we observed few soft tissue-related complications such as union-related problems $(0 \%)$, deep infection $(0 \%)$, and other soft tissue complications $(2 \%)$. Lateral tibial plateau fracture during opening-wedge high tibial osteotomy is uncommon, but it is a severe complication when it occurs. Marti et al. [25] reported a $3 \%$ of incidence in series of 323 segments. We experienced no tibial plateau fracture in our case series. We believe the proximal half pin, which is inserted deeply up to $1 \mathrm{~cm}$ medial to the lateral tibial cortex, can help prevent accidental fracture of the lateral tibial plateau when applying valgus force.

The fixator-assisted technique showed advantages in controlling alignment in fracture and/or deformity correction surgeries [6-8, 22]. Our study showed that this fixatorassisted technique achieved accurate target correction in the coronal plane while maintaining posterior tibial slope in the sagittal plane. Conventional opening-wedge high tibial osteotomy has several technical pitfalls that can result in increased posterior tibial slope, internal torsion of the tibia, etc $[14,15,24]$. Although the tibial slope is important in knee kinematics and proper function of the knee ligaments, maintaining constant tibial slope after opening-wedge high 
tibial osteotomy still remains an unsolved problems $[1,11$, 24, 27]. Several surgical methods were recommended to avoid an increase in posterior tibial slope in conventional opening-wedge high tibial osteotomy: sufficient release of posterior soft tissues, complete osteotomy of the posterior tibial cortex, avoidance of an intact cortical hinge on the posterolateral side, and full extended position of the knee during fixation with plating, all of which showed limitations [5, 27, 29]. In our technique, we applied various surgical considerations to prevent an increase in posterior tibial slope: posteriorly located half pins, positioning of the spreader at the posteromedial corner of the osteotomy gap, and pushing down on the knee to make the knee fully extended during plating. In the current study, the mean absolute difference between pre- and postoperative posterior proximal tibial angle was less than $1^{\circ}$ in all patients $(100 \%)$. Among these surgical considerations, we believe that the posteriorly located half pins for temporary external fixation provided strong resistant force against an increase in the posterior tibial slope during the surgery. Changes in tibial rotation in opening-wedge high tibial osteotomy and how to control it have not been fully investigated in the literature. Jacobi et al. [14] demonstrated significant changes in tibial rotation $\left(>5^{\circ}\right)$ occurred in $11.9 \%$ in a cadaver model. This fixator-assisted technique can also have another benefit in controlling tibial rotation during surgery, because the two half pins for temporary external fixation can be used to maintain or even intentionally create a certain amount of rotation.

We found that less invasive plating through the fixatorassisted technique in opening-wedge high tibial osteotomy has the following two benefits: (1) the less invasive technique showed low risk of nonunion, deep infection, soft tissue complications, and minimized accidental fracture of the lateral tibial plateau; and (2) the fixator-assisted technique provided easy control of coronal and sagittal alignment and showed the ability to control rotational alignment as well.

In conclusion, the authors' technique is a valid option for medial opening-wedge osteotomy, which enables less invasive surgery with relatively accurate coronal/sagittal/ rotational alignment control. However, future studies should compare this approach with other approaches for proximal tibial osteotomy to ascertain whether indeed this procedure is less invasive and more reliable. Several disadvantages of this fixator-assisted technique should be considered carefully. It requires additional surgical time and more radiation exposure to the operating surgeon to ensure proper location of the half pins. However, easy control of the alignment and the firm temporary fixation during plating can help reduce the overall surgical time for experienced surgeons.

\section{References}

1. Bombaci H, Canbora K, Onur G, Görgeç M. The effect of open wedge osteotomy on the posterior tibial slope. Acta Orthop Traumatol Turc. 2005;39:404-410.

2. Brosset T, Pasquier G, Migaud H, Gougeon F. Opening wedge high tibial osteotomy performed without filling the defect but with locking plate fixation (TomoFix ${ }^{\mathrm{TM}}$ ) and early weight-bearing: prospective evaluation of bone union, precision and maintenance of correction in 51 cases. Orthop Traumatol Surg Res. 2011;97:705-711.

3. Collinge CA, Sanders RW. Percutaneous plating in the lower extremity. J Am Acad Orthop Surg. 2000;8:211-216.

4. Cotic M, Vogt S, Hinterwimmer S, Feucht MJ, Slotta-Huspenina J, Schuster T, Imhoff AB. A matched-pair comparison of two different locking plates for valgus-producing medial open-wedge high tibial osteotomy: peek-carbon composite plate versus titanium plate. Knee Surg Sports Traumatol Arthrosc. 2014 Feb 22 [Epub ahead of print].

5. Ducat A, Sariali E, Lebel B, Mertl P, Hernigou P, Flecher X, Zayni R, Bonnin M, Jalil R, Amzallag J, Rosset P, Servien E, Gaudot F, Judet T, Catonné Y. Posterior tibial slope changes after opening- and closing-wedge high tibial osteotomy: a comparative prospective multicenter study. Orthop Traumatol Surg Res. 2012;98:68-74.

6. Eidelman M, Keren Y, Norman D. Correction of distal femoral valgus deformities in adolescents and young adults using minimally invasive fixator-assisted locking plating (FALP). $J$ Pediatr Orthop B. 2012;21:558-562.

7. El-Rosasy MA, El-Sallakh SA. Distal tibial hypertrophic nonunion with deformity: treatment by fixator-assisted acute deformity correction and LCP fixation. Strategies Trauma Limb Reconstr. 2013;8:31-35.

8. Fitoussi F, Ilharreborde B, Lefevre Y, Souchet P, Presedo A, Mazda K, Penneçot GF. Fixator-assisted medial tibial plateau elevation to treat severe Blount's disease: outcomes at maturity. Orthop Traumatol Surg Res. 2011;97:172-178.

9. Floerkemeier S, Staubli AE, Schroeter S, Goldhahn S, Lobenhoffer P. Outcome after high tibial open-wedge osteotomy: a retrospective evaluation of 533 patients. Knee Surg Sports Traumatol Arthrosc. 2013;21:170-180.

10. Fujusawa Y, Masuhara K, Shiomi S. The effect of high tibial osteotomy on osteoarthritis of the knee. Orthop Clin North Am. 1979;10:585-608.

11. Giffin JR, Vogrin TM, Zantop T, Woo SLY, Harner CD. Effects of increasing tibial slope on the biomechanics of the knee. Am J Sports Med. 2004;32:376-382.

12. Han SB, Bae JH, Lee SJ, Jung TG, Kim KH, Kwon JH, Nha KW. Biomechanical properties of a new anatomical locking metal block plate for opening wedge high tibial osteotomy: uniplane osteotomy. Knee Surg Relat Res. 2014;26:155-161.

13. Han SB, Lee DH, Shetty GM, Chae DJ, Song JG, Nha KW. A 'safe zone' in medial open-wedge high tibia osteotomy to prevent lateral cortex fracture. Knee Surg Sports Traumatol Arthrosc. 2013;21:90-95.

14. Jacobi M, Villa V, Reischl N, Demey G, Goy D, Neyret P, Gautier E, Magnussen RA. Factors influencing posterior tibial slope and tibial rotation in opening wedge high tibial osteotomy. Knee Surg Sports Traumatol Arthrosc. 2014 Jun 11 [Epub ahead of print].

15. Jung WH, Chun CW, Lee JH, Ha JH, Kim JH, Jeong JH. Comparative study of medial opening-wedge high tibial osteotomy using 2 different implants. J Arthrosc Relat Surg. 2013;29:1063-1071.

16. Kim JH, Kim JR, Lee DH, Bang JY, Hong IT. Combined medial open-wedge high tibial osteotomy and modified Maquet 
procedure for medial compartmental osteoarthritis and patellofemoral arthritis of the knee. Eur J Orthop Surg Traumatol. 2013; 23:679-683.

17. Kim JW, Oh CW, Jung WJ, Kim JS. Minimally invasive plate osteosynthesis for open fractures of the proximal tibia. Clin Orthop Surg. 2012;4:313-320.

18. Kocaoglu M, Eralp L, Bilen FE, Balci HI. Fixator-assisted acute femoral deformity correction and consecutive lengthening over an intramedullary nail. J Bone Joint Surg Am. 2009;91:152-159.

19. Kolb W, Guhlmann H, Windisch C, Marx F, Kolb K, Koller H. Fixation of distal femoral fractures with the Less Invasive Stabilization System: a minimally invasive treatment with locked fixed-angle screws. J Trauma. 2008;65:1425-1434.

20. Krettek C, Schandelmaier P, Miclau T, Bertram R, Holmes W, Tscherne $\mathrm{H}$. Transarticular joint reconstruction and indirect plate osteosynthesis for complex distal supracondylar femoral fractures. Injury. 1997;28(Suppl 1):A31-41.

21. Krettek C, Schandelmaier P, Miclau T, Tscherne H. Minimally invasive percutaneous plate osteosynthesis (MIPPO) using the DCS in proximal and distal femoral fractures. Injury. 1997; 28(Suppl 1):A20-30.

22. Lee HJ, Oh CW, Oh JK, Apivatthakakul T, Kim JW, Yoon JP, Lee DJ, Jung JW. Minimally invasive plate osteosynthesis for humeral shaft fracture: a reproducible technique with the assistance of an external fixator. Arch Orthop Trauma Surg. 2013;133:649-657.
23. Lobenhoffer $\mathrm{P}$, Agneskirchner JD. Improvements in surgical technique of valgus high tibial osteotomy. Knee Surg Sports Traumatol Arthrosc. 2003;11:132-138.

24. Marti CB, Gautier E, Wachtl SW, Jakob RP. Accuracy of frontal and sagittal plane correction in open-wedge high tibial osteotomy. Arthroscopy. 2004;20:366-372.

25. Martin R, Birmingham TB, Willits K, Litchfield R, Lebel M-E, Giffin JR. Adverse event rates and classifications in medial opening wedge high tibial osteotomy. Am J Sports Med. 2014; 42:1118-1126.

26. Meidinger $\mathrm{G}$, Imhoff $\mathrm{AB}$, Paul J, Kirchhoff $\mathrm{C}$, Sauerschnig M, Hinterwimmer S. May smokers and overweight patients be treated with a medial open-wedge HTO? Risk factors for nonunion. Knee Surg Sports Traumatol Arthrosc. 2011;19:333-339.

27. Ozalay M, Ozkoc G, Circi E, Akpinar S, Hersekli MA, Uysal M, Cesur N. The correlation of correction magnitude and tibial slope changes following open wedge high tibial osteotomy. Knee Surg Sports Traumatol Arthrosc. 2008;16:948-951.

28. Ronga M, Shanmugam C, Longo UG, Oliva F, Maffulli N. Minimally invasive osteosynthesis of distal tibial fractures using locking plates. Orthop Clin North Am. 2009;40:499-504.

29. Wang JH, Bae JH, Lim HC, Shon WY, Kim CW, Cho JW. Medial open wedge high tibial osteotomy: the effect of the cortical hinge on posterior tibial slope. Am J Sports Med. 2009; 37:2411-2418. 